\title{
Tetramethylpyrazine alleviates endoplasmic reticulum stress-activated apoptosis and related inflammation in chondrocytes
}

\author{
SHUAI HU ${ }^{1 *}$, SHENG WANG $^{1 *}$, JIE HE $^{1}$ and YANGYANG BIAN ${ }^{2}$ \\ ${ }^{1}$ Joint and Traumatology Department; ${ }^{2}$ Department of Trauma Medical Center, \\ The First Affiliated Hospital of Hainan Medical University, Haikou, Hainan 570100, P.R. China
}

Received May 22, 2021; Accepted September 7, 2021

DOI: $10.3892 / \mathrm{mmr} .2021 .12528$

\begin{abstract}
Excessive apoptosis of chondrocytes and degradation of the extracellular matrix (ECM) contribute to the typical pathological characteristics of osteoarthritis (OA). Various studies have reported that tetramethylpyrazine (TMP) protects against multiple disorders by inhibiting inflammation and oxidative stress. The present study investigated the effects of TMP on chondrocytes and evaluated the associated mechanisms. To determine the effect of TMP on OA and the underlying mechanisms, chondrocytes were incubated with TMP and IL- $1 \beta$ or thapsigargin (TG) Western blotting assays were performed to examine the expression levels of endoplasmic reticulum (ER) stress proteins, and TUNEL staining, fluorescence immunostaining and reverse transcription-quantitative PCR were used to determine the apoptosis levels, and catabolic and inflammatory factors. It was found that TMP protected chondrocytes by suppressing IL- $1 \beta$-induced expression of glucose-regulated protein 78 (GRP78) and CHOP (an apoptotic protein). TMP regulated the TG-mediated upregulated expression of GRP78 and CHOP in the chondrocytes of rats, as well as markedly suppressed levels of ER stress-triggered inflammatory cytokines (TNF- $\alpha$ and IL-6). Furthermore, TMP modulated TG-induced changes in ECM catabolic metabolism in rat chondrocytes. Collectively, TMP alleviated ER-stress-activated apoptosis and related inflammation in chondrocytes, indicating that it has therapeutic potential for the treatment of OA.
\end{abstract}

Correspondence to: Professor Yangyang Bian, Department of Trauma Medical Center, The First Affiliated Hospital of Hainan Medical University, 31 Longhua Road, Longhua, Haikou, Hainan 570100, P.R. China

E-mail: yangbianbian2018@126.com

*Contributed equally

Key words: tetramethylpyrazine, endoplasmic reticulum stress, osteoarthritis, apoptosis, inflammation

\section{Introduction}

During the onset and development of osteoarthritis (OA), chondrocytes are altered via exposure to pro-inflammatory cytokines (1-3). In addition, the upregulation of pro-inflammatory cytokines, such as IL- $1 \beta$, in the cartilage and serum of patients with OA have been associated with OA development (4). IL-1 $\beta$ can promote degradation of the extracellular matrix (ECM) by enhancing the generation of catabolic enzymes, such as MMPs, resulting in cartilage disruption $(5,6)$. The reduction of IL-1 $\beta$ levels was previously proposed as an anti-OA therapeutic strategy (7). When OA is caused by the upregulation of IL-1 $\beta$, the NF- $\kappa B$ signal transduction pathway is presumably activated, which could regulate the production of apoptotic and inflammatory proteins (8).

The apoptosis of chondrocytes is a primary cause of cartilage disruption $(9,10)$. Endoplasmic reticulum (ER) stress, which is caused by glucose or oxygen deprivation, certain therapeutic agents [e.g. thapsigargin (TG)] and free radical damage, can induce the activation of apoptotic pathways $(11,12)$. Although the ER can initially protect against stress caused by misfolded protein accumulation by maintaining cell homeostasis, a severe unfolded protein response (UPR) eventually reduces this protection $(13,14)$. The upregulated expression of protein disulfide isomerase and glucose-regulated protein 78 (GRP78), two markers of ER stress, is observed in OA articular cartilage, indicating that ER stress is associated with OA development (15).

Tetramethylpyrazine (TMP), also known as ligustrazine, is a nitrogenous heterocyclic compound comprising of one carbon atom in a pyrazine ring attached to one methyl group (16). TMP is the major active alkaloid ingredient in Chinese herbal medicines (Ligusticum wallichii) and can dilate blood vessels, inhibit platelet aggregation and improve microcirculation. Accordingly, it has been extensively used in clinical settings (17). TMP ameliorates corneal neovascularization by regulating cell infiltration into the cornea after alkali burn (16). TMP also exerts therapeutic effects on sepsis-induced acute lung injury in rats by inhibiting ER stress (18). TMP can also limit ER stress resulting from placental gestational diabetes mellitus (19). However, to the best of our knowledge, no studies have examined the effects of 
TMP for the treatment of OA, and few studies have assessed the potential mechanism of TMP in cartilage degeneration. To address these issues, the current study aimed to investigate the protective effect of TMP on ER stress, inflammation and oxidative stress in OA chondrocytes. Moreover, the effects of TMP on ECM metabolism and the degree of apoptosis, as well as the mechanisms underlying the effects of IL-1 $\beta$ exposure in chondrocytes were evaluated.

\section{Materials and methods}

Reagents. TMP (purity $>98 \%$ ) and TG, a compound frequently used to induce ER stress, were provided by Sigma-Aldrich (Merck KGaA). A caspase 3 activity kit (cat. no. K186-100) was purchased from BioVision, Inc. and primary antibodies [CHOP (cat. no. 2895; 1:1,000) and GRP78 (cat. no. 3183; 1:1,000)] were provided by Cell Signaling Technology, Inc. Antibody against collagen (Col) II (cat. no. sc-52658; 1:50) was obtained from Santa Cruz Biotechnology, Inc. Additionally, an in-situ cell death detection kit (cat. no. 11684817910) was provided by Roche Diagnostics. The remaining reagents were provided by Sigma-Aldrich (Merck KGaA), except as otherwise noted.

Cell culture and drug treatment. Each animal experiment was performed in accordance with the guidelines of the Animal Care and Use Committee of Hainan Medical University and was approved by the Animal Care and Use Committee of Hainan Medical College (approval no. 2019-23). Chondrocytes were isolated and cultured as previously described (20). The experiments were carried out between January 2019 and November 2020. In total, 30 3-month-old male SpragueDawley rats (Animal Center of the Chinese Academy of Sciences; weight, 250-300 g) were used. All rats were housed under specific pathogen-free laboratory conditions $\left(18-29^{\circ} \mathrm{C}\right.$; $40-70 \%$ humidity), with free access to food and water. After animals had been sacrificed via $\mathrm{CO}_{2}$ inhalation $(40 \% \mathrm{vol} / \mathrm{min}$ for $5 \mathrm{~min}$ ), their knee-joint cartilage was removed under aseptic conditions and the tissue was cut using micro-scissors. Then, $0.25 \%(\mathrm{w} / \mathrm{v})$ trypsin-ethylenediaminetetraacetic acid was used to treat the cartilage fragments at $37^{\circ} \mathrm{C}$ for $30 \mathrm{~min}$, followed by treatment with $0.2 \%(\mathrm{w} / \mathrm{v})$ collagenase II in serum-free DMEM (Gibco; Thermo Fisher Scientific, Inc.) at $37^{\circ} \mathrm{C}$ for $4 \mathrm{~h}$. The fragments were resuspended and filtered using a $150-\mu \mathrm{m}$ mesh. The obtained chondrocytes were plated into high-glucose (4.5 g/l) DMEM that was supplemented with 10\% FBS (Gibco; Thermo Fisher Scientific, Inc.) and 1\% antibiotics (streptomycin/penicillin) at $5 \times 10^{6}$ cells $/ 25 \mathrm{~cm}^{2}$ flask, then incubated at $37^{\circ} \mathrm{C}(21)$. Chondrocytes from passage 2 (Fig. S1) were used for cell experiments.

Cells were incubated with TMP $(100 \mu \mathrm{M})(22,23)$ at $37^{\circ} \mathrm{C}$ for $24 \mathrm{~h}$ in the presence or absence of TG $(10 \mu \mathrm{M})(24)$. The concentrations of TMP and TG were mainly selected based on our preliminary experiments and previous reports as aforementioned.

Western blotting assay. RIPA lysis buffer (Sigma-Aldrich; Merck KGaA) containing protease and phosphatase inhibitors was used to homogenize the chondrocyte culture, and a BCA protein assay kit was used to assess the protein content. Next, $8-10 \%$ SDS-PAGE was performed to resolve the proteins
(50 $\mu \mathrm{g})$, followed by transfer onto nitrocellulose membranes. Membranes were then incubated in 5\% skim milk powder at room temperature for $90 \mathrm{~min}$, followed by incubation with a primary antibody overnight at $4{ }^{\circ} \mathrm{C}$ and then with secondary antibody [anti-mouse IgG (cat. no. 7076; 1:1,000; Cell Signaling Technology, Inc.) or anti-rabbit IgG (cat. no. 7074; 1:1,000; Cell Signaling Technology, Inc.)] for $60 \mathrm{~min}$ at room temperature. Finally, protein bands were detected using a western blotting detection reagent (cat. no. 32106; Thermo Fisher Scientific, Inc.) under an imaging system (Bio-Rad Laboratories, Inc.). Densitometric analysis was performed using Image Lab 3.0 software (Bio-Rad Laboratories, Inc.).

Apoptosis analysis. Caspase 3 and TUNEL kits were used to detect apoptotic activity. In brief, chondrocytes were subjected to incubation in $4 \%$ paraformaldehyde for $1 \mathrm{~h}, 3 \%(\mathrm{v} / \mathrm{v}) \mathrm{H}_{2} \mathrm{O}_{2}$ for $15 \mathrm{~min}$ and $0.1 \%$ Triton $\mathrm{X}-100$ for $8 \mathrm{~min}$, both at room temperature. Thereafter, an in-situ cell death detection kit was used to analyze the specimens at room temperature, and nuclei were visualized via DAPI staining for $8 \mathrm{~min}$ at room temperature. The samples were examined using a Nikon Eclipse Ti confocal microscope (Nikon Corporation). A total of 20-30 visual fields (magnification, $\mathrm{x} 40$ ) in each group were analyzed to count the apoptotic cells.

Apoptotic activity was evaluated using a Caspase 3 Activity Assay kit in accordance with the manufacturer's instructions.

Immunofluorescence assay. After drug treatment, cells were subjected to incubation in $4 \%$ paraformaldehyde for $1 \mathrm{~h}, 0.5 \%$ Triton X-100 for 8 min and 5\% BSA (Thermo Fisher Scientific, Inc.) for $45 \mathrm{~min}$, all at room temperature. Next, an anti-Col II (1:50) primary antibody was incubated with the cells overnight at $4^{\circ} \mathrm{C}$, followed by another $1 \mathrm{~h}$ incubation with a secondary antibody (mouse IgG - H\&L Alexa Fluor ${ }^{\circledR} 488$; 1:200; cat. no. ab150113; Abcam) at room temperature. Subsequently, cells were incubated with DAPI at room temperature for $8 \mathrm{~min}$ to enable visualization of nuclei. Finally, a Nikon Eclipse Ti confocal microscope (magnification, $\mathrm{x} 40$; Nikon Corporation) was used to examine samples.

Reverse transcription-quantitative (RT-q)PCR. Total RNA was obtained from chondrocyte samples using TRIzol ${ }^{\circledR}$ (Invitrogen; Thermo Fisher Scientific, Inc.) in accordance with the manufacturer's instructions. RNA was quantified via spectrophotometry. In total, 500 ng RNA was reverse transcribed and amplified using the PrimeScript-RT reagent kit and SYBR Premix Ex Taq mixture (Takara Bio, Inc.), respectively. The following thermocycling conditions were used for the qPCR: Initial denaturation for $10 \mathrm{~min}$ at $95^{\circ} \mathrm{C}$, followed by 40 cycles for $15 \mathrm{sec}$ at $95^{\circ} \mathrm{C}$ and $1 \mathrm{~min}$ at $60^{\circ} \mathrm{C}$. The $2^{-\Delta \Delta \mathrm{Cq}}$ method was used to calculate expression of the target gene (25). The level of target mRNA was normalized to the level of GAPDH. The following primer sequences were used: COX-2 forward, 5'-CCAGAGCAGAGAGATGAAATACCA-3' and reverse, 5'-GCAGGGCGGGATACAGTTC-3'; inducible nitric oxide synthase forward, 5'-GATTCAGTGGTCCAACCTGCA-3' and reverse, 5'-CGACCTGATGTTGCCACTGTT-3'; IL-1 $\beta$ forward, 5'-CCCTGCAGCTGGAGAGTGTGG-3' and reverse, 5'-TGTGCTCTGCTTGAGAGGTGCT-3'; IL-6 forward, 5'-CGAGCCCACCAGGAACGAAAGTC-3' and 
B

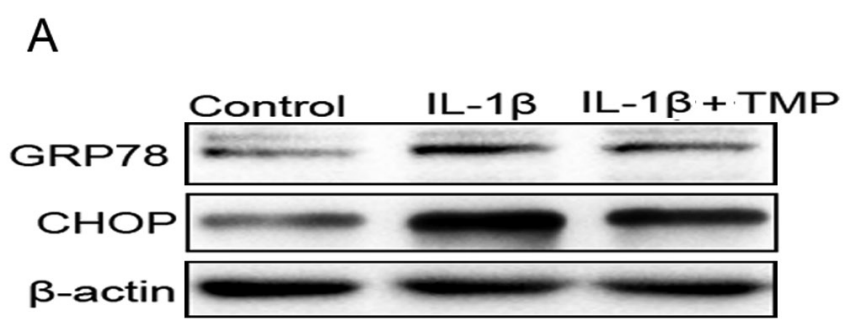

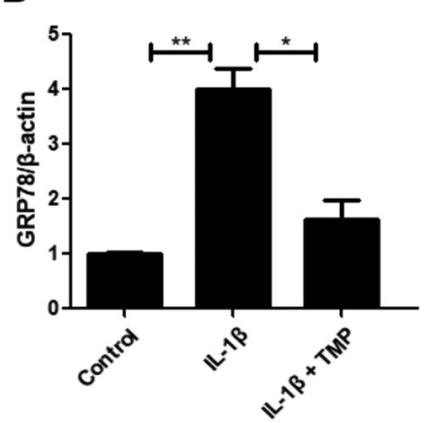

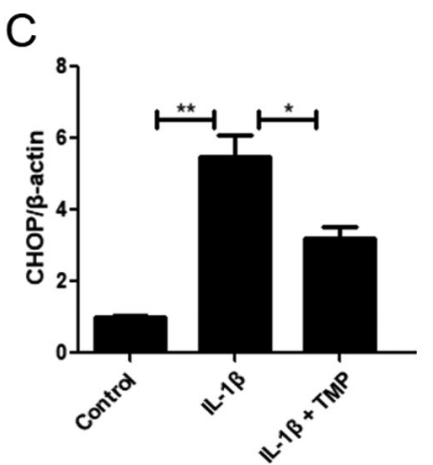

Figure 1. TMP mitigates endoplasmic reticulum stress and subsequent apoptosis of IL-1 1 -exposed chondrocytes. (A) Western blot analysis of (B) GRP78 and (C) CHOP expression in IL-1 $\beta$-treated chondrocytes. Data are presented as the mean $\pm \mathrm{SD}$. " $\mathrm{P}<0.05,{ }^{* *} \mathrm{P}<0.01$. GRP78, glucose-regulated protein 78 ; TMP, tetramethylpyrazine.

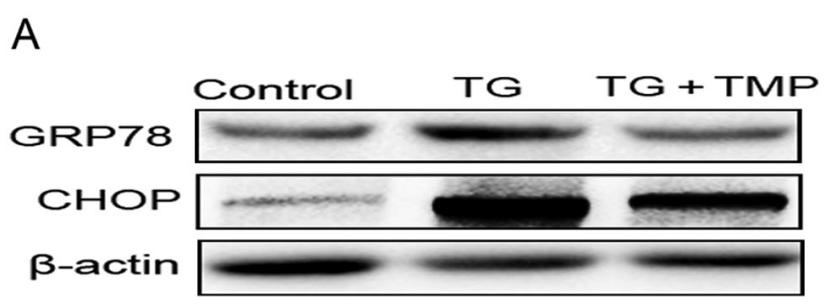

B

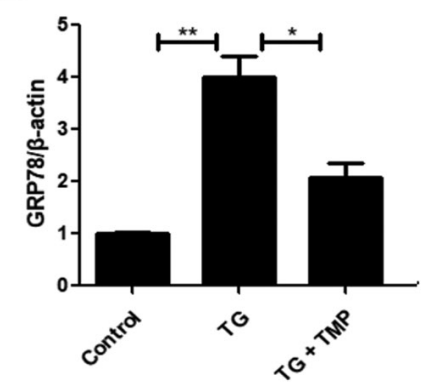

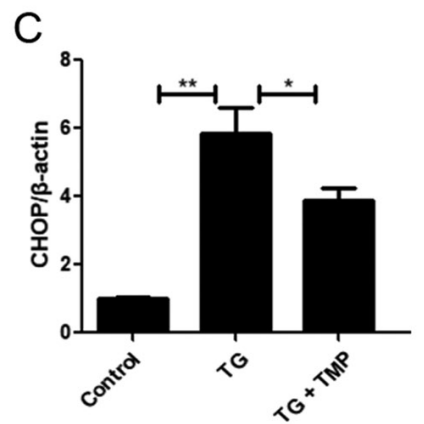

Figure 2. TMP suppresses TG-mediated ER stress and subsequent expression of apoptotic proteins in chondrocytes. (A) Western blot analysis of (B) GRP78 and (C) CHOP expression in TG-treated chondrocytes. Data are presented as the mean $\pm \mathrm{SD}$. ${ }^{*} \mathrm{P}<0.05,{ }^{* *} \mathrm{P}<0.01$. GRP78, glucose-regulated protein 78 ; TMP, tetramethylpyrazine; TG, thapsigargin.

reverse, 5'-CTGGCTGGAAGTCTCTTGCGGAG-3'; TNF- $\alpha$ forward, 5'-GAAGCCCCTCCCAGTTCTAGTTC-3' and reverse, 5'-CACTCCCCATCCTCCCTGGTC-3'; МMP-3 forward, 5'-TCCCTGTTCAGCCATCCCTTG-3' and reverse, 5'-TCGCTCTGGTAGCCCTTCTC-3'; МMP-13 forward, 5'-TGGTCCCTGCCCCTTCCCTA-3' and reverse, 5'-CCGCAAGAGTCACAGGATGGTAGTA-3'; thrombospondin type 1 motif 4 (ADAMTS-4) forward, 5'-GGA ATGGTGGAAAGTATTGTGAGG-3' and reverse, 5'-GAGGTCGGTTCGGTGGTTGT-3'; thrombospondin type 1 motif 5 (ADAMTS-5) forward, 5'-CAACTTGACATTTGGGCCTGA-3' and reverse, 5'-TCCACGGCAGGCAACTTCT-3'; and GAPDH forward, 5'-AACGGCACAGTCAAGGCTGA-3' and reverse, 5'-ACGCCAGTAGACTCCACGACAT-3'.

Statistical analysis. Statistical analysis was performed using SPSS version 19.0 software (IBM Corp.). The results are presented as the mean \pm SD. Differences between groups were assessed using one-way ANOVA, followed by the Tukey's post hoc test. Each experiment was repeated at least five times. $\mathrm{P}<0.05$ was considered to indicate a statistically significant difference.

\section{Results}

TMP alleviates ER stress and subsequent apoptosis of $I L-1 \beta$-treated chondrocytes. ER stress plays an important role in the pathological mechanism of OA. In this process, UPR sensors separate from the chaperone protein GRP78 and are then phosphorylated, which contributes to activation of $\mathrm{CHOP}$ and subsequent apoptosis $(26,27)$. To determine the sensitivity of ER stress to TMP exposure in IL-1 $\beta$-exposed chondrocytes, the levels of ER stress-related factors, including GRP78 and CHOP (downstream apoptotic protein), were assessed. Western blot analysis revealed that the aforementioned proteins were upregulated upon IL-1 $\beta$ exposure, while TMP had the opposite effects (Fig. 1).

TMP inhibits TG-induced ER stress and subsequent expression of apoptotic proteins in chondrocytes. In order to clarify whether the cytoprotective effects of TMP were associated with its regulation of ER stress, chondrocytes were treated with TG, a commonly used inducer of ER stress. Western blot analysis indicated that compared with controls, the expression levels of CHOP and GRP78 were upregulated in TG-exposed chondrocytes. Furthermore, TMP treatment partly abolished this upregulated CHOP and GRP78 expression (Fig. 2A-C). Collectively, these findings indicated that TMP markedly attenuated ER stress level in OA chondrocytes.

TMP inhibits TG-induced apoptosis in vitro. To further investigate the relationship between ER stress and the role of TMP in resisting apoptosis in TG-treated chondrocytes, a caspase 3 activity assay and TUNEL staining of chondro- 


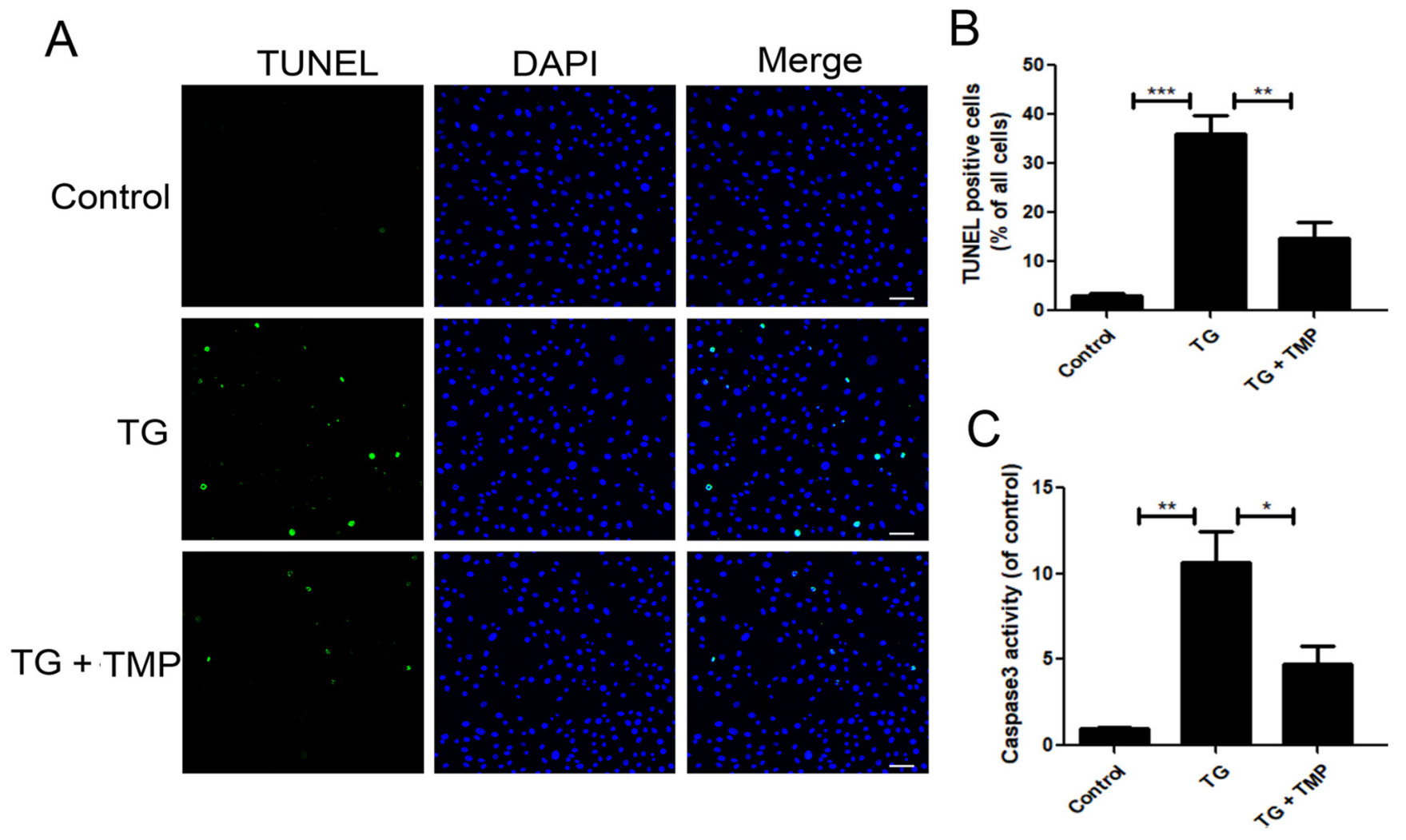

Figure 3. TMP suppresses TG-mediated apoptosis in vitro. (A) A TUNEL assay was used to detect apoptosis (scale bar, $50 \mu \mathrm{m}$ ), (B) and quantification of results. (C) Caspase 3 activities of chondrocytes in different conditions. Data are expressed as the mean $\pm \mathrm{SD}$. ${ }^{*} \mathrm{P}<0.05,{ }^{* *} \mathrm{P}<0.01,{ }^{* * *} \mathrm{P}<0.001$. TMP, tetramethylpyrazine; TG, thapsigargin.

cytes were conducted. TUNEL staining identified that TMP significantly reduced the number of apoptotic cells among TG-treated chondrocytes (Fig. 3A and B). The results of the caspase 3 activity assay were consistent with the suppression of TG-mediated apoptosis upon TMP treatment (Fig. 3C). Collectively, these findings indicated that the anti-apoptotic effects of TMP were mediated by the suppression of ER stress.

TMP suppresses the mRNA expression levels of inflammatory cytokines induced by TG in chondrocytes. Next, RT-qPCR analysis was conducted to examine the mRNA expression levels of inflammatory factors (including inducible nitric oxide synthase, IL- 6 , IL-1 $\beta$, cyclooxygenase- 2 and TNF- $\alpha$ ). Upregulated expression of inflammatory factors (IL-6, IL-1 $\beta$, iNOS, COX-2 and TNF- $\alpha$ ) were observed in TG-exposed chondrocytes.

TMP suppresses TG-activated catabolic activity in chondrocytes. Catabolic enzymes, including MMP-3 and -13, as well as ADAM metallopeptidase with thrombospondin type 1 motif 4 (ADAMTS-4) and -5 , are closely associated with ECM decomposition in chondrocytes (5). As shown in Fig. 5, the expression levels of ADAMTS-4 and -5 were markedly increased in the TG group, and this pattern was reversed by TMP. Similarly, TMP significantly decreased the enhanced expression levels of MMP-3 and -13 in the TG group. These findings suggested that TMP exposure suppressed TG-mediated chondrocyte catabolism.
TMP suppresses TG-activated ECM degradation in chondrocytes. Immunofluorescence and PCR assays were performed to measure ECM degradation-associated mRNAs and proteins. The expression levels of aggrecan and Col II, the two main ECM components, were significantly downregulated after TG exposure (Fig. 6A and B). Nonetheless, TMP-exposed chondrocytes exhibited increased mRNA expression levels of aggrecan and Col II, compared with TG-exposed cells. Immunofluorescence staining for Col II showed results similar to those of PCR analysis (Fig. 6C). These findings indicated that TMP exposure protected ECM proteins within TG-treated nucleus pulposus cells.

\section{Discussion}

Cartilage contains chondrocytes and ECM, which comprise a small portion of the overall tissue volume. However, chondrocytes have been implicated in the generation and maintenance of ECM proteins (e.g., Cols II, IX and $\mathrm{XI}$ ), proteoglycans (i.e., aggrecan) and glycoproteins (5). Mutations of genes that encode cartilage components can trigger skeletal dysplasia and potentially impair the production of cartilage components, leading to the accumulation of protein aggregates within the ER. Cartilage damage is induced by mutant variants in the ER, rather than a lack of certain ECM proteins (15). Such mutated ECM components can aggregate and destroy chondrocyte homeostasis, by means of ER stress, thereby promoting the pathogenic mechanisms of chondrodysplasias (28). In the 
A IL-1 $\beta$

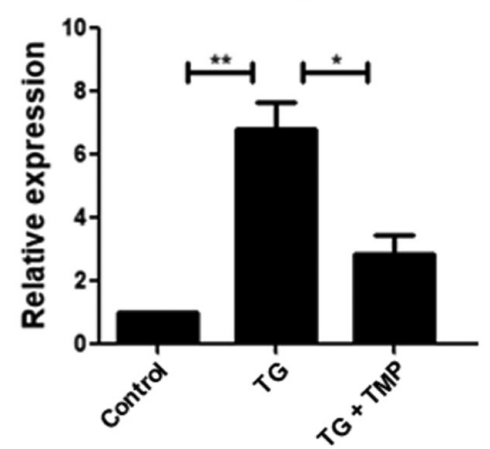

D

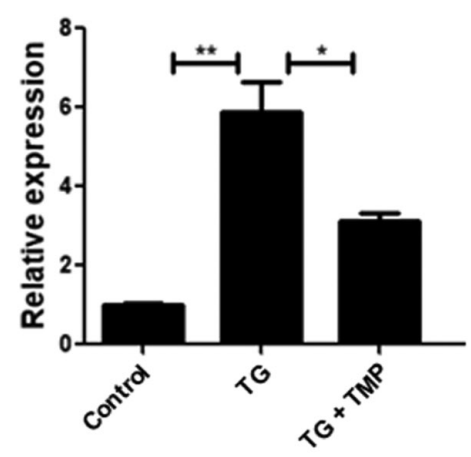

IL-6

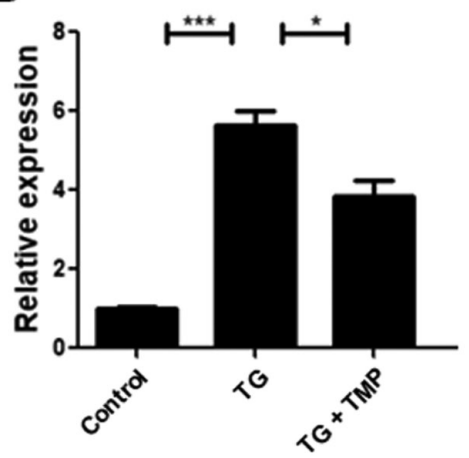

E

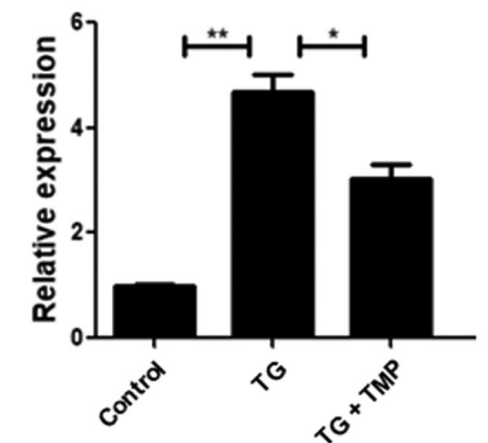

C

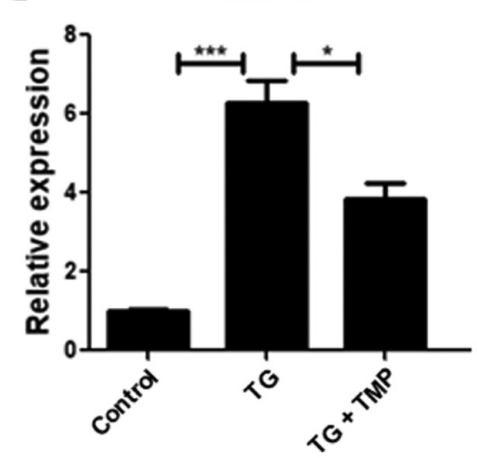

Figure 4. TMP suppresses the mRNA expression levels of inflammatory cytokines induced by TG in chondrocytes. Relative mRNA expression levels of (A) IL-1 $\beta$, (B) IL-6, (C) TNF- $\alpha$, (D) COX-2 and (E) iNOS detected via reverse transcription-quantitative PCR. Data are expressed as the mean \pm SD. "P<0.05, ${ }^{* *} \mathrm{P}<0.01,{ }^{* * *} \mathrm{P}<0.001$. COX-2, cyclooxygenase-2; iNOS, inducible nitric oxide synthase; TMP, tetramethylpyrazine; TG, thapsigargin.

A

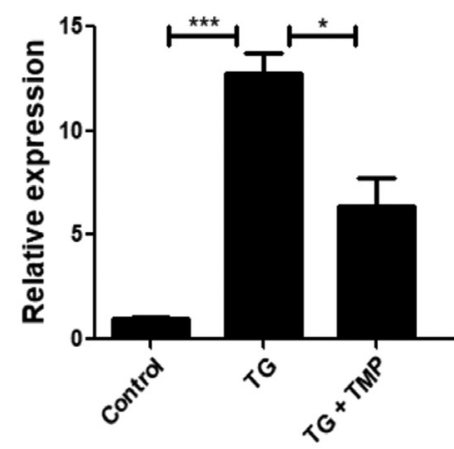

C

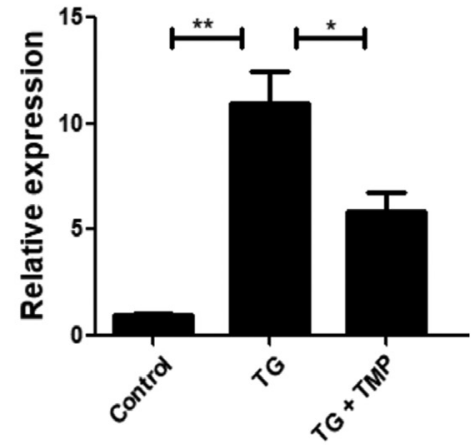

B

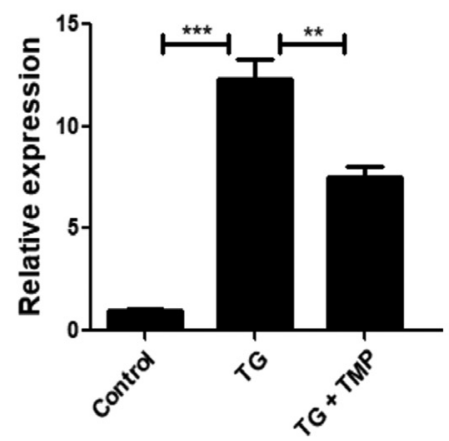

D

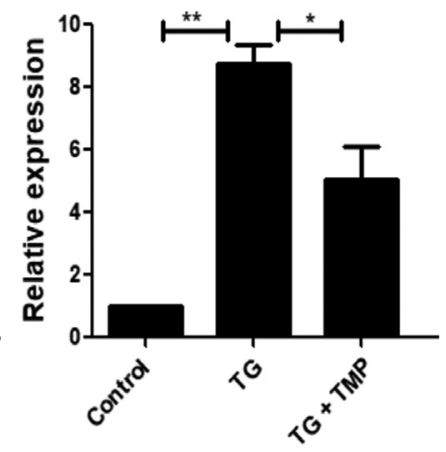

Figure 5. TMP suppresses TG-activated catabolic activity in chondrocytes. Relative mRNA expression levels of (A) MMP-3, (B) MMP-13, (C) ADAMTS-4 and (D) ADAMTS-5 detected via reverse transcription-quantitative PCR. Data are expressed as the mean $\pm \mathrm{SD}$. ${ }^{*} \mathrm{P}<0.05,{ }^{* *} \mathrm{P}<0.01,{ }^{* * *} \mathrm{P}<0.001$. TMP, tetramethylpyrazine; TG, thapsigargin; ADAMTS, ADAM metallopeptidase with thrombospondin type 1 motif. 

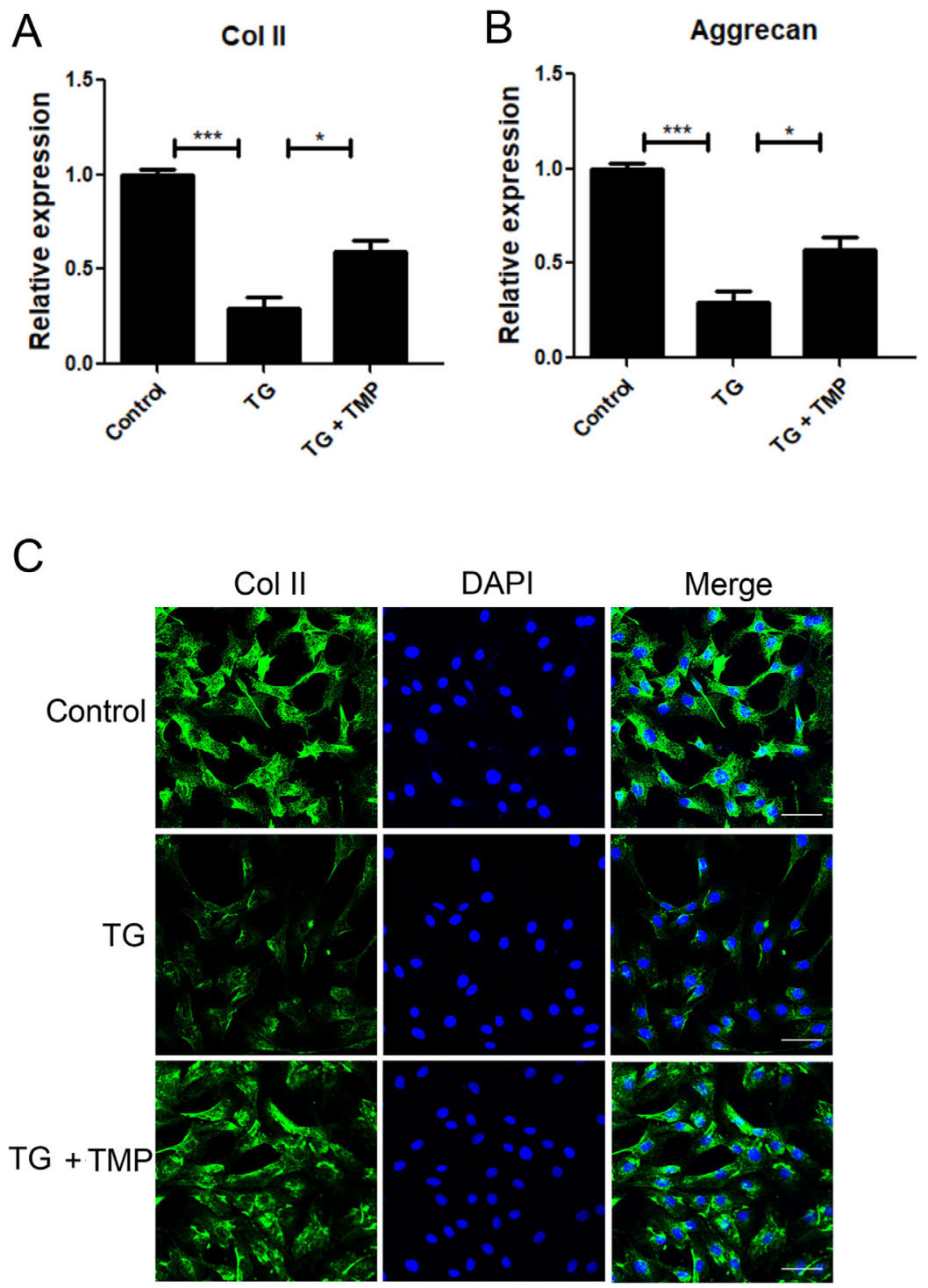

Figure 6. TMP suppresses TG-activated ECM degradation in chondrocytes. Relative mRNA expression levels of (A) Col II and (B) aggrecan were detected via reverse transcription-quantitative PCR. (C) Immunofluorescence staining for Col II proteins (scale bar, $50 \mu \mathrm{m}$ ). Data are expressed as the mean $\pm \mathrm{SD}$. " $\mathrm{P}<0.05$, ${ }^{* * * *} \mathrm{P}<0.001$. Col, collagen; TMP, tetramethylpyrazine; TG, thapsigargin.

ColIITgcog mouse model, the expression of thyroglobulin (the mutated thyroid protein) within chondrocytes indicates that ER stress can sufficiently induce a short stature disorder, similar to chondrodysplasia (29). Irrespective of the presence or absence of mutated thyroglobulin, ER stress is aggravated by destabilization of the medial meniscus-mediated OA induction within chondrocytes. Typically, susceptibility to ER stress in the ColIITgcog mouse model has a beneficial effect. Specifically, it can mitigate OA due to the effective management of ER stress within articular chondrocytes (30).

Certain stress sensors can recognize misfolded proteins, thus initiating the UPR. For this reason, all cells contain the necessary components, such as activating transcription factor 4 , activating transcription factor $6 \alpha$, GRP78, protein kinase RNA-like ER kinase, inositol-required enzyme $1 \alpha$, CHOP and X-box binding protein 1 (31). When CHOP transcription is initiated, apoptosis is induced in response to severe ER stress, thus activating death effectors, such as Bim and Bcl-2 $(32,33)$. Reversal of $\mathrm{Ca}^{2+}$-activated $\mathrm{K}^{+}$channel suppression by inhibition of ER stress-induced $\beta 1$ subunit loss can protect TMP from the impact of homocysteine on coronary dilation (34). In a gestational diabetes mellitus mouse model, TMP can mitigate placental oxidative stress, inflammation and ER stress (35). Furthermore, TMP alleviates sepsis-triggered acute lung injury by inhibiting the apoptosis of pulmonary microvascular endothelial cells via the protein kinase RNA-like ER kinase/ eIF2a/activating transcription factor 4/CHOP apoptosis signal transduction pathway during ER stress (18). The results of the present study indicated that TMP has a protective effect on chondrocytes, a possible relationship with ER stress and a role in apoptosis signal transduction. The current results demonstrated that TMP treatment decreased CHOP and GRP78 expression levels in cells exposed to IL-1 $\beta$, suggesting a possible mechanism for TMP signaling. To optimize the investigation into the function of ER stress in OA, the present study used TG, which is known to induce ER stress in in vitro models (24). It was found that TMP exposure markedly decreased TG-triggered ER stress within chondrocytes. Moreover, exposure to TMP 
partly abolished TG-induced chondrocyte apoptosis. These results indicated that ER stress was closely associated with the protection of TMP in chondrocytes.

The aggravation of OA over time may be attributed to decreased ER folding- and UPR-associated protein activities and expression (36). Older adults are more likely to have ER stress at the ECM protein synthesis stage, which can resemble the initial stage of OA (37). The present study found that TG-exposed chondrocytes showed characteristics of cartilage degeneration, such as downregulation of ECM proteins (aggrecan and Col II) and upregulation of degrading enzymes (MMP-3 and -13, and ADAMTS-4 and -5) secreted by chondrocytes, although TMP partly abolished these effects.

Inflammation contributes to the variability in OA characteristics, and may be associated with both joint symptoms and OA deterioration $(38,39)$. The upregulation of matrix-degrading enzymes and pro-inflammatory factors can destroy cartilage and enhance chondrocyte senescence, thereby promoting OA deterioration (40). The upregulation of inflammatory cytokines (e.g., TNF- $\alpha$ and IL-1 $\beta$ ) can increase the expression levels of catabolic proteins, such as MMPs and ADAMTS, thereby accelerating the progressive losses of proteoglycans and Cols $(41,42)$. IL-1 $\beta$ is widely applied in studies of the pathophysiology of OA to trigger the release of inflammatory cytokines, as well as chondrocyte apoptosis (6). Regulation of the levels of several catabolic enzymes (MMPs or ADAMTS) is achieved by transcription factors, especially NF- $\mathrm{NB}(7,8)$.

In an OA rat model, p65 knockdown suppressed disease deterioration at the early stage (43). TMP mitigates endotoxin-mediated retinal inflammation by suppressing the activation of microglial cells via the Toll-like receptor $4 / \mathrm{NF}-\kappa \mathrm{B}$ signal transduction pathway (44). TMP also decreases inflammation in liver fibrosis, while suppressing the production of inflammatory cytokines within hepatic stellate cells via regulation of the NLR family pyrin domain containing 3 inflammasome pathway (45). The current findings indicated that the mechanisms underlying the effects of TMP on TG-exposed chondrocytes included the inhibition of inflammatory mediators. The TG-exposed chondrocytes showed inflammatory characteristics, while TMP markedly reversed these effects.

However, the present study was not without its limitations. First, no further animal experiments were conducted on the effect of TMP on OA, and should be performed in further studies. Second, this work did not evaluate the effect of TMP on the upstream pathway related to ER stress. Further research should be performed to reveal the pharmacological mechanisms of TMP in OA.

In conclusion, the present study demonstrated that TMP enhanced cell viability, reduced inflammatory cytokine production and suppressed apoptosis. Such protection was associated with TMP-mediated ER stress regulation within chondrocytes. Further investigations are required to determine whether TMP has potential as a therapeutic agent for the prevention of cartilage destruction in patients with $\mathrm{OA}$.

\section{Acknowledgements}

Not applicable.

\section{Funding}

No funding was received.

\section{Availability of data and materials}

The datasets used and/or analyzed during the current study are available from the corresponding author on reasonable request.

\section{Authors' contributions}

SH and YB designed the study. JH and SH contributed to statistical analysis, data interpretation and manuscript preparation. SW and SH performed the experiments and interpretation. $\mathrm{SH}$ and YB confirm the authenticity of all the raw data. All authors read and approved the final manuscript.

\section{Ethics approval and consent to participate}

The present study was approved by the Animal Care and Use Committee of Hainan Medical College.

\section{Patient consent for publication}

Not applicable.

\section{Competing interests}

The authors declare that they have no competing interests.

\section{References}

1. Goldring MB: Chondrogenesis, chondrocyte differentiation, and articular cartilage metabolism in health and osteoarthritis. Ther Adv Musculoskelet Dis 4: 269-285, 2012.

2. Suri S and Walsh DA: Osteochondral alterations in osteoarthritis. Bone 51: 204-211, 2012.

3. Sui C, Zhang $\mathrm{L}$ and $\mathrm{Hu} \mathrm{Y}$ : MicroRNA-let-7a inhibition inhibits LPS-induced inflammatory injury of chondrocytes by targeting IL6R. Mol Med Rep 20: 2633-2640, 2019.

4. Liu-Bryan R and Terkeltaub R: Emerging regulators of the inflammatory process in osteoarthritis. Nat Rev Rheumatol 11: 35-44, 2015.

5. Bondeson J, Wainwright S, Hughes C and Caterson B: The regulation of the ADAMTS4 and ADAMTS5 aggrecanases in osteoarthritis: A review. Clin Exp Rheumatol 26: 139-145, 2008.

6. Lefebvre V, Peeters-Joris C and Vaes G: Modulation by interleukin 1 and tumor necrosis factor alpha of production of collagenase, tissue inhibitor of metalloproteinases and collagen types in differentiated and dedifferentiated articular chondrocytes. Biochim Biophys Acta 1052: 366-378, 1990.

7. Tang S, Tang Q, Jin J, Zheng G, Xu J, Huang W, Li X, Shang P and Liu H: Polydatin inhibits the IL-1 $\beta$-induced inflammatory response in human osteoarthritic chondrocytes by activating the Nrf2 signaling pathway and ameliorates murine osteoarthritis. Food Funct 9: 1701-1712, 2018

8. Feng Z, Li X, Lin J, Zheng W, Hu Z, Xuan J, Ni W and Pan X: Oleuropein inhibits the IL-1 $\beta$-induced expression of inflammatory mediators by suppressing the activation of NF- $\kappa \mathrm{B}$ and MAPKs in human osteoarthritis chondrocytes. Food Funct 8: 3737-3744, 2017

9. Sharif M, Whitehouse A, Sharman P, Perry M and Adams M: Increased apoptosis in human osteoarthritic cartilage corresponds to reduced cell density and expression of caspase- 3 . Arthritis Rheum 50: 507-515, 2004.

10. Zou Y, Liu Q, Guo P, Huang Y, Ye Z and Hu J: Anti-chondrocyte apoptosis effect of genistein in treating inflammation-induced osteoarthritis. Mol Med Rep 22: 2032-2042, 2020. 
11. Cláudio N, Dalet A, Gatti E and Pierre P: Mapping the crossroads of immune activation and cellular stress response pathways. EMBO J 32: 1214-1224, 2013.

12. Wictome M, Henderson I, Lee AG and East JM: Mechanism of inhibition of the calcium pump of sarcoplasmic reticulum by thapsigargin. Biochem J 283: 525-529, 1992.

13. Bernales S, Papa FR and Walter P: Intracellular signaling by the unfolded protein response. Annu Rev Cell Dev Biol 22: 487-508, 2006.

14. Hetz C: The unfolded protein response: Controlling cell fate decisions under ER stress and beyond. Nat Rev Mol Cell Biol 13: 89-102, 2012

15. Nugent AE, Speicher DM, Gradisar I, McBurney DL, Baraga A, Doane KJ and Horton WE Jr: Advanced osteoarthritis in humans is associated with altered collagen VI expression and upregulation of ER-stress markers Grp78 and bag-1. J Histochem Cytochem 57: 923-931, 2009.

16. Wu Y, Xu Z, Yang Y, Qiu J, Yang M, Wu C, Lai Z, Tang M, Ge J, Yu K, et al: Tetramethylpyrazine (TMP) ameliorates corneal neovascularization via regulating cell infiltration into cornea after alkali burn. Biomed Pharmacother 109: 1041-1051, 2019.

17. Zhao Y, Liu Y and Chen K: Mechanisms and clinical application of tetramethylpyrazine (an interesting natural compound isolated from Ligusticum wallichii): Current status and perspective. Oxid Med Cell Longev 2016: 2124638, 2016.

18. Liu W, Liu K, Zhang S, Shan L and Tang J: Tetramethylpyrazine showed therapeutic effects on sepsis-induced acute lung injury in rats by inhibiting endoplasmic reticulum stress protein kinase RNA-like endoplasmic reticulum kinase (PERK) signalinginduced apoptosis of pulmonary microvascular endothelial cells. Med Sci Monit 24: 1225-1231, 2018.

19. Yung HW, Alnæs-Katjavivi P, Jones CJ, El-Bacha T, Golic M, Staff AC and Burton GJ: Placental endoplasmic reticulum stress in gestational diabetes: The potential for therapeutic intervention with chemical chaperones and antioxidants. Diabetologia 59: 2240-2250, 2016.

20. Jiang LB, Lee S, Wang Y, Xu QT, Meng DH and Zhang J: Adipose-derived stem cells induce autophagic activation and inhibit catabolic response to pro-inflammatory cytokines in rat chondrocytes. Osteoarthritis Cartilage 24: 1071-1081, 2016.

21. Xue EX, Lin JP, Zhang Y, Sheng SR, Liu HX, Zhou YL and $\mathrm{Xu}$ H: Pterostilbene inhibits inflammation and ROS production in chondrocytes by activating Nrf2 pathway. Oncotarget 8 : 41988-42000, 2017.

22. Muralidharan P, Acosta MF, Gomez AI, Grijalva C, Tang H, Yuan JXJ and Mansour HM: Design and comprehensive characterization of tetramethylpyrazine (TMP) for targeted lung delivery as inhalation aerosols in pulmonary hypertension $(\mathrm{PH})$ In vitro human lung cell culture and in vivo efficacy. Antioxidants (Basel) 10: 427, 2021.

23. Fang Y, Chu L, Li L, Wang J, Yang Y, Gu J and Zhang J: Tetramethylpyrazine protects bone marrow-derived mesenchymal stem cells against hydrogen peroxide-induced apoptosis through PI3K/Akt and ERK1/2 pathways. Biol Pharm Bull 40: 2146-2152, 2017

24. Liu D, Gu Y, Wang W and Chen W: Astragalin alleviates ischemia/reperfusion-induced brain injury via suppression of endoplasmic reticulum stress. Mol Med Rep 22: 4070-4078, 2020.

25. Pfaffl MW: A new mathematical model for relative quantification in real-time RT-PCR. Nucleic Acids Res 29: e45, 2001.

26. Feng K, Ge Y, Chen Z, Li X, Liu Z, Li X, Li H, Tang T, Yang F and Wang X: Curcumin inhibits the PERK-eIF2 $\alpha$-CHOP pathway through promoting SIRT1 expression in oxidative stress-induced rat chondrocytes and ameliorates osteoarthritis progression in a rat model. Oxid Med Cell Longev 2019: 8574386, 2019.

27. Husa M, Petursson F, Lotz M, Terkeltaub R and Liu-Bryan R: $\mathrm{C} / \mathrm{EBP}$ homologous protein drives pro-catabolic responses in chondrocytes. Arthritis Res Ther 15: R218, 2013

28. Kung LH, Rajpar MH, Briggs MD and Boot-Handford RP: Hypertrophic chondrocytes have a limited capacity to cope with increases in endoplasmic reticulum stress without triggering the unfolded protein response. J Histochem Cytochem 60: 734-748, 2012.
29. Rajpar MH, McDermott B, Kung L, Eardley R, Knowles L, Heeran M, Thornton DJ, Wilson R, Bateman JF, Poulsom R, et al: Targeted induction of endoplasmic reticulum stress induces cartilage pathology. PLoS Genet 5: e1000691, 2009.

30. Kung LHW, Mullan L, Soul J, Wang P, Mori K, Bateman JF, Briggs MD and Boot-Handford RP: Cartilage endoplasmic reticulum stress may influence the onset but not the progression of experimental osteoarthritis. Arthritis Res Ther 21: 206, 2019.

31. Hetz C, Zhang K and Kaufman RJ: Mechanisms, regulation and functions of the unfolded protein response. Nat Rev Mol Cell Biol 21: 421-438, 2020.

32. Oyadomari S, Koizumi A, Takeda K, Gotoh T, Akira S, Araki E and Mori M: Targeted disruption of the Chop gene delays endoplasmic reticulum stress-mediated diabetes. J Clin Invest 109: 525-532, 2002

33. Puthalakath H, O'Reilly LA, Gunn P, Lee L, Kelly PN, Huntington ND, Hughes PD, Michalak EM, McKimmBreschkin J, Motoyama N, et al: ER stress triggers apoptosis by activating BH3-only protein Bim. Cell 129: 1337-1349, 2007.

34. Sun WT, Wang XC, Novakovic A, Wang J, He GW and Yang Q: Protection of dilator function of coronary arteries from homocysteine by tetramethylpyrazine: Role of ER stress in modulation of BKCa channels. Vascul Pharmacol 113: 27-37, 2019.

35. Jiao Y, Zhang S, Zhang J and Du J: Tetramethylpyrazine attenuates placental oxidative stress, inflammatory responses and endoplasmic reticulum stress in a mouse model of gestational diabetes mellitus. Arch Pharm Res 42: 1092-1100, 2019.

36. Brown MK and Naidoo N: The endoplasmic reticulum stress response in aging and age-related diseases. Front Physiol 3: 263 , 2012.

37. Venn G, Billingham ME and Hardingham TE: Increased proteoglycan synthesis in cartilage in experimental canine osteoarthritis does not reflect a permanent change in chondrocyte phenotype. Arthritis Rheum 38: 525-532, 1995.

38. Zheng G, Zhan Y, Tang Q, Chen T, Zheng F, Wang H, Wang J, Wu D, Li X, Zhou Y, et al: Monascin inhibits IL-1 $\beta$ induced catabolism in mouse chondrocytes and ameliorates murine osteoarthritis. Food Funct 9: 1454-1464, 2018.

39. Jia J, Sun J, Liao W, Qin L, Su K, He Y, Zhang J, Yang R, Zhang Z and Sun Y: Knockdown of long non-coding RNA AK094629 attenuates the interleukin-1 $\beta$ induced expression of interleukin- 6 in synovium-derived mesenchymal stem cells from the temporomandibular joint. Mol Med Rep 22: 1195-1204, 2020.

40. Greene MA and Loeser RF: Aging-related inflammation in osteoarthritis. Osteoarthritis Cartilage 23: 1966-1971, 2015.

41. Irie K, Uchiyama E and Iwaso H: Intraarticular inflammatory cytokines in acute anterior cruciate ligament injured knee. Knee 10: $93-96,2003$

42. Speziali A, Delcogliano M, Tei M, Placella G, Chillemi M, Tiribuzi R and Cerulli G: Chondropenia: Current concept review. Musculoskelet Surg 99: 189-200, 2015.

43. Csaki C, Mobasheri A and Shakibaei M: Synergistic chondroprotective effects of curcumin and resveratrol in human articular chondrocytes: Inhibition of IL-1beta-induced NF-kappaBmediated inflammation and apoptosis. Arthritis Res Ther 11: R165, 2009.

44. Hu PF, Sun FF, Jiang LF, Bao JP and Wu LD: Paeoniflorin inhibits IL-1 $\beta$-induced MMP secretion via the NF- $\mathrm{B}$ p pathway in chondrocytes. Exp Ther Med 16: 1513-1519, 2018.

45. Zhuang Z, Ye G and Huang B: Kaempferol alleviates the interleukin-1 $\beta$-induced inflammation in rat osteoarthritis

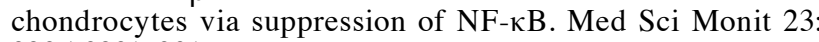
3925-3931, 2017.

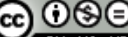

This work is licensed under a Creative Commons Attribution-NonCommercial-NoDerivatives 4.0 International (CC BY-NC-ND 4.0) License. 